\title{
Percutaneous vertebroplasty versus percutaneous kyphoplasty for the treatment of delayed post-traumatic vertebral body collapse (Kümmell's disease) in Chinese patients: a systematic review and meta-analysis ${ }^{\dagger}$
}

Original article

Peng-Fei Hana,\#, Cheng-Long Chen",\#, Tao-Yu Chen ${ }^{\mathrm{b}}$, Zhi-Liang Zhang ${ }^{\mathrm{b}}$, Xiao-Dong Lib, Peng-Cui Li ${ }^{b}$, Xiao-Chun Wei ${ }^{b, *}$

aDepartment of Orthopaedic Surgery, The Second People’s Hospital of Changzhi, Changzhi, Shanxi 046000, China

${ }^{b}$ Department of Orthopaedic Surgery, The Second Hospital of Shanxi Medical University, Taiyuan, Shanxi 030009, China

Received: 25 January 2019; Accepted: 6 March 2018; Published: 20 December 2019

Abstract: Objective: To compare the clinical efficacy between percutaneous vertebroplasty (PVP) and percutaneous kyphoplasty (PKP) in the treatment of Kümmell's disease in Chinese patients.

Methods: The studies using randomized controlled trials to compare clinical efficacy between PVP and PKP in the treatment of Kümmell's disease in Chinese patients were retrieved from Embase, Pubmed, Central, Cinahl, PQDT, CNKI, CQVIP, Wanfang Data, and CBM (from September 2008 to September 2018). Keywords for both Chinese and English search were: percutaneous vertebroplasty, PVP, percutaneous kyphoplasty, PKP, and Kümmell's disease. A total of 132 articles were retrieved based on the search strategy through online database searching and manual searching. Finally, one foreign report and seven Chinese reports were included. After extracting the data, statistical software Review Manager 5.3 was used for data analysis.

Results: Through comparison, Cobb angle $(95 \% \mathrm{Cl}[0.54,4.42), P=0.01]$ and Oswestry Dysfunction Index (ODI) $(95 \% \mathrm{Cl}[0.21$, 2.15], $P=0.02$ ) of PKP group was smaller than that of PVP group. Postoperative anterior vertebral body height of the PKP group was better than PVP group $(95 \% \mathrm{Cl}[-1.27,-0.66], P<0.001]$. However, the PVP group had shorter operation time than PKP group (95\% $\mathrm{Cl}[-13.48,-7.43), P=0.001]$. In the other outcome measures, including Visual Analogue Scale (VAS) score $(95 \% \mathrm{Cl}$ $[-0.04,0.27), P=0.15)$, cement volume $(95 \% \mathrm{Cl}[-0.82,0.32], P=0.39)$ and cement leakage $(95 \% \mathrm{Cl}[0.90,2.76], P=0.11)$, there was no significant differences between the two procedures.

Conclusions: At this stage, there is sufficient evidence to support that PKP is better than PVP in the treatment of Kümmell's disease in Chinese patients. Although PVP surgery requires much less operation time, PKP has better postoperative radiological results and lower ODI. Moreover, both of them had similar clinical results (e.g., analgesic effects, cement dosage, and leakage rate). Further evidence is dependent on the emergence of randomized controlled trials with higher quality and larger sample sizes in the future.

Keywords: Kümmell's disease • percutaneous vertebroplasty • percutaneous kyphoplasty • post-trauma • vertebral body collapse - meta-analysis • systematic review

(c) Shanxi Medical Periodical Press.

\#Peng-Fei Han and Cheng-Long Chen were considered as co-first authors.

${ }^{\dagger}$ This project was supported by National Project of International Science and technology Cooperation program of China (No. 2015DFA33050), National Natural Science Foundation of China for Youths (No. 81601949).

How to cite this article: Han PF, Chen CL, Chen TY, et al. Percutaneous vertebroplasty versus percutaneous kyphoplasty for the treatment of delayed post-traumatic vertebral body collapse (Kümmell's disease) in Chinese patients: a systematic review and meta-analysis. Front Nurs. 2019; 4: 273-284.

ठ Open Access. ๑ 2019 Peng-Fei Han et al., published by Sciendo. (c) Bryc-ND This work is licensed under the Creative Commons Attribution NonCommercial-NoDerivatives 4.0 License. 


\section{Introduction}

Kümmell's disease, otherwise known as delayed posttraumatic vertebral collapse and avascular necrosis of the vertebral body, after a mild traumatic experience in an asymptomatic period, was first described by the German surgeon Kümmell in 1895..$^{1,2}$ Due to the rare and complicated conditions, Kümmell's disease has so far been reported few times. Kümmell's disease causes only mild symptoms at an early stage, and the duration of the disease varies, which can delay diagnosis and treatment. ${ }^{3}$ In the late stage, vertebral body collapse and kyphotic deformity are caused, which often leads to severe intractable back pain. ${ }^{4}$ Some patients even have symptoms of spinal cord compression, which severely impacts the quality of life. Kümmell's disease treatment includes conservative and surgical methods, which includes PVP, PKP minimally invasive surgery, and open surgery. As the vertebral body of Kümmell's patients is destroyed and does not heal itself, conservative treatment is often ineffective for Kümmell's disease and requires surgical intervention. ${ }^{5}$ Surgical treatment of patients with Kümmell's disease results in better clinical outcomes such as good pain relief, functional improvement, and kyphosis correction. Both the PVP and PKP are minimally invasive surgery, which percutaneously injected cement into the vertebral body under local anesthesia and X-ray to enhance the strength and stability of the vertebral body. ${ }^{6}$ Percutaneous vertebroplasty (PVP) and kyphoplasty can effectively relieve fracture pain and even partially restore the height of vertebral body. At the same time, the thermal effect generated by polymerization of bone cement can further alleviate the pain of nerve degeneration and necrosis in and around vertebral body. Therefore, the operation has the advantages of simple operation, exact curative effect, small trauma, and early activity of the patient. ${ }^{7,8}$

Currently, it is generally believed that PVP surgery is more economical than PKP surgery because there are no consumables such as balloons. Clinical application has confirmed that PVP is a safe and effective surgical procedure;, 10 PKP is a better choice for patients with significant vertebral height loss and old fractures with pseudo articular formation. PKP can better restore vertebral height and correct kyphosis. ${ }^{11,12}$ On the other hand, due to the injection of bone cement into the compressed vertebral body under a large pressure, both types of minimally invasive surgery may cause complications such as leakage of bone cement. Reportedly, both procedures can be used for the treatment of Kümmell's disease, but the optimal choice between these two procedures remains controversial. ${ }^{13,14}$ This metaanalysis intends to systematically compare the efficacy between the two procedures in order to provide some theoretical guidance for clinical practice.

\section{Materials and methods}

\subsection{Search strategy}

The retrieval was conducted in the online databases include Embase, Pubmed, Central, Cinahl, PQDT, CNKI, CQVIP, Wanfang Data, Cochrane Library, and CBM. We also manually searched some journals' cata$\log$ and references, and strive to find gray literature, such as unpublished academic papers and chapters in monographs. Searching all relevant papers without restricting the language and translating if necessary. Keywords searched for both Chinese and English were: Percutaneous Vertebroplasty, PVP, Percutaneous Kyphoplasty, PKP, and Kümmell's disease. Search strategy was: Kümmell's disease AND "Percutaneous Vertebroplasty OR PVP" AND "Percutaneous Kyphoplasty OR PKP."

\subsection{Inclusion criteria}

Inclusion criteria for the analysis were (1) Chinese adults with phase I and II Kümmell's disease; (2) randomized controlled trials (RCTs), prospective studies, retrospective studies, and cohort studies; (3) Patients received minimally invasive surgery for the treatment of Kümmell's disease; (4) study compared results of PVP and PKP, (5) The outcome being measured by Cobb angle, anterior vertebral body height, Oswestry Dysfunction Index (ODI), Visual Analogue Scale (VAS) score, cement volume, cement leakage, and operation time.

\subsection{Exclusion criteria}

The exclusion criteria were: (1) letters, comments, editorials, case reports, proceedings, personal communications, or reviews; (2) study objective or intervention measures failed to meet the inclusion criteria; (3) the original documents of experimental design being not precise; and (4) studied with incomplete data.

\subsection{Data extraction and quality assessment}

Inclusion decisions were made independently by two reviewers participated in according to the pre-stated eligiblity criteria. Disagreement between the two reviewers was resolved by discussion or consulting to a third reviewer when necessary. The risk-of-bias assessment tool outlined in Cochrane Handbook was used to measure the methodological quality of case-controlled trials (CCTs). Six domains are evaluated: random sequence generation, allocation concealment, blinding of patients and personal, blinding of outcome assessment, incomplete outcome data, and selective reporting risk. 
The Newcastle-Ottawa Scale (NOS) was used to assess the quality of cohort studies, the full score is 9 points. Trials with a score of more than 6 points are considered high-quality study. Relevant data were recorded in this analysis, including: first author's name, published year, sample size of PVP and PKP in the treatment of Kümmell's disease, and so on.

\subsection{Statistical analysis}

Data were independently entered into RevMan 5.3 software by two reviewers. Dichotomous outcomes were expressed in terms of Odds ratio (OR) and the weighted mean difference (WMD) or the standard mean difference (SMD) was used for continuous outcomes, both with $95 \%$ confidence intervals $(95 \% \mathrm{Cl})$. Heterogeneity was tested using both the chi-square test and $I^{2}$ test. A fixed-effects model was chosen when there was no statistical evidence of heterogeneity $\left(I^{2}<50 \%\right)$ and random-effects model was adopted if significant heterogeneity was found. If the heterogeneity was found, we checked the study population, treatment, outcome, and methodologies to determine the source of heterogeneity. If it could not be quantitatively synthesized or the event rate was too low to measure, we used qualitative evaluation. By eliminating some of the studies for sensitivity analysis and making funnel plots to assess the bias. The difference was considered statistically significant when $P \leq 0.05$.

\section{Results}

Based on the above mentioned search strategy, a total of 132 related articles were retrieved. By reading the titles and abstracts, we excluded 55 noncontrolled studies, repeated publications, and 40 articles that are not related to the research purpose. Thirty-seven related studies were screened out preliminarily; further reading the full texts and the screening criteria were strictly followed according to the inclusion and exclusion criteria. Finally, one English article and seven Chinese articles were included. Patient's characteristics and conditions in included study were compared such as age, gender, and so on. The differences were not significant $(P>0.05)$. The literature screening process and the results are shown in Figure 1. Basic characteristics of included literature are indicated in Table 1.

\subsection{Cobb angle}

To compare preoperative and postoperative Cobb angle changes in PVP and PKP groups of Chinese Kümmell patients, five clinical studies were included. These studies were divided into three subgroups based on preoperative, postoperative 1-2 days and last follow-up. Random effect model was employed in metaanalysis because the heterogeneity between the studies and subgroups was significant $\left(I^{2}>50 \%\right)$. The results showed that PKP group had less Cobb angle than PVP group 1-2 days after operation $(95 \% \mathrm{Cl}[0.91,8.36]$, $P=0.01)$, but preoperative Cobb angle $(95 \% \mathrm{Cl}[-1.25$, 1.05], $P=0.86)$ and the last follow-up Cobb angle $(95 \%$ $\mathrm{Cl}[-0.76,6.41], P=0.12)$ was similar, and the difference was not statistically significant (Figure 2).

\subsection{Anterior vertebral body height}

According to the preoperative, 1-2 days after operation, and the last follow-up, data were divided into three subgroups. A total of six trials were included, and preoperative and postoperative vertebral body height of Chinese Kümmell's patients between PVP and PKP groups were compared. Random effect model was employed in metaanalysis because the heterogeneity between the studies and subgroups was significant $\left(I^{2}>50 \%\right)$. The results showed that the height of anterior vertebral body in the PKP group was better than PVP group on 1-2 days postoperative $(95 \% \mathrm{Cl}[-5.49,-0.26], P=0.03)$ and the last follow-up (95\% Cl $[-4.7,-0.29], P=0.03)$, and the difference was statistically significant (Figure 3 ).

\subsection{Oswestry Dysfunction Index}

According to the preoperative, 1-2 days after operation, and the last follow-up, data were divided into three subgroups. A total of six trials were included, and preoperative and postoperative ODI of Chinese Kümmell's patients in PVP and PKP groups were compared. Random effect model was employed in meta-analysis because the heterogeneity between the studies and subgroups was significant $\left(R^{2}>50 \%\right)$. The results showed that PKP group had less ODI score than PVP group 1-2 days after operation $(95 \% \mathrm{Cl}[0.00,1.81]$, $P=0.05)$, but preoperative ODI score $(95 \% \mathrm{Cl}[-0.41$, 3.84], $P=0.11)$ and the last follow-up ODI score $(95 \%$ $\mathrm{Cl}[-0.60,3.46], P=0.17]$ was similar, and the difference was not statistically significant (Figure 4).

\subsection{VAS score}

According to the preoperative, 1-2 days after operation, and the last follow-up, data were divided into three subgroups. A total of eight trials were included, and preoperative and postoperative VAS score of Chinese Kümmell's patients between PVP and PKP groups was compared. Random effect model was employed in meta-analysis because the heterogeneity between the studies and subgroups was significant $\left(R^{2}>50 \%\right)$. 
Records identified through database searching $(n=129)$
Additional records identified through other sources $(n=3)$

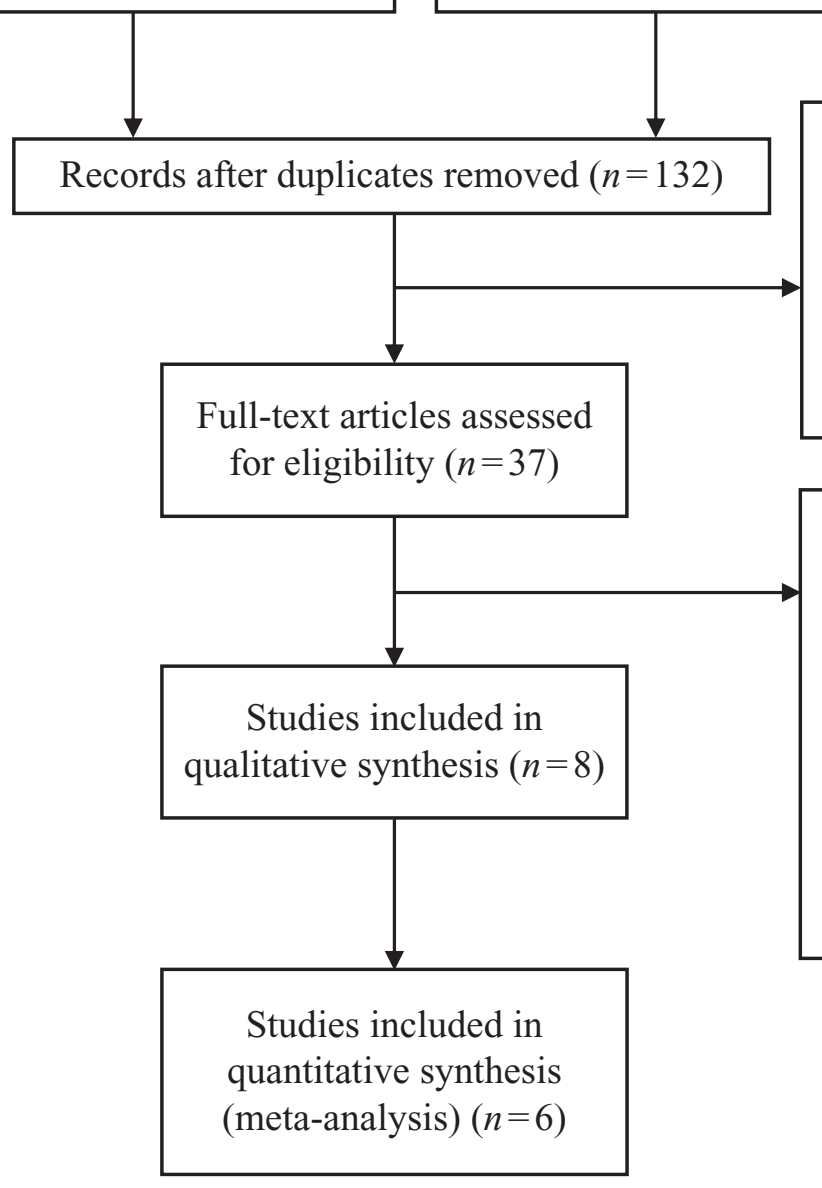

Records excluded:

- Duplicates $(n=3)$;

- Systematic review or meta-analysis $(n=3)$;

- Unrelated articles $(n=89)$;

Full-text articles excluded with reasons:

- Not case controlled study $(n=16)$;

- Uncorrelated operative methods $(n=13)$;

- Not case controlled study $(n=12)$;

Figure 1. Flow diagram of search strategy.

The results showed that in PVP group and PKP group, preoperative VAS score $(95 \% \mathrm{Cl}[-0.09,0.45], P=0.19)$, VAS score at $1-2$ days after operation $(95 \% \mathrm{Cl}[-0.20$, $0.15], P=0.79)$ and the last follow-up VAS score $(95 \%$ $\mathrm{Cl}[-0.18,0.52], P=0.35)$ was similar, and the difference was not statistically significant (Figure 5).

\subsection{Cement volume}

For 6 studies included we compared the amounts of cement injected between PVP and PKP procedures. Random effect model was employed in meta-analysis because the heterogeneity between the studies was significant $\left(I^{2}>50 \%\right)$. The meta-analysis showed that amounts of cement injected in PVP and PKP procedures were similar $(95 \% \mathrm{Cl}[-0.82,0.32), P=0.39)$, there was no significant difference between the two groups (Figure 6).

\subsection{Cement leakage}

Seven studies included have compared the cases of cement leakage of PVP and PKP procedures. Fixed effect model was employed in meta-analysis with the absence of heterogeneity $\left(R^{2}<50 \%\right)$ among the seven study results. The meta-analysis showed that the occurrence of cement was similar between the two groups (95\% Cl [0.90, 2.76], $P=0.11$ ), and the difference was not statistically significant (Figure 7 ).

\subsection{Operation time}

Five trials included have compared the operation time between PVP and PKP procedures. Random effect model was employed in meta-analysis because the heterogeneity between the studies was significant $\left(R^{2}>50 \%\right)$. The meta-analysis showed that the operation 


\begin{tabular}{|c|c|c|c|c|c|c|c|}
\hline Author & Study design & Group & Cases & Age (y) & Gender (M/F) & Outcomes & Newcastle-Ottawa Scale \\
\hline \multirow{2}{*}{$\begin{array}{l}\text { Chen et al. } \\
2012^{15}\end{array}$} & \multirow{2}{*}{ Retrospective } & PVP & 33 & $69.2 \pm 6.3$ & $4 / 29$ & \multirow{2}{*}{$D, E, F, G$} & \multirow{2}{*}{$\star \star \star \star \star$} \\
\hline & & PKP & 30 & $68.7 \pm 6.5$ & $3 / 27$ & & \\
\hline \multirow{2}{*}{$\begin{array}{l}\text { Feng and Sun } \\
2018^{16}\end{array}$} & \multirow{2}{*}{ Retrospective } & PVP & 20 & \multirow{2}{*}{$72.3 \pm 5.4$} & \multirow{2}{*}{$12 / 28$} & \multirow{2}{*}{$A, B, C, D, E, F, G$} & \multirow{2}{*}{$\star \star \star \star \star \star$} \\
\hline & & PKP & 20 & & & & \\
\hline \multirow{2}{*}{$\begin{array}{l}\text { Gao et al. } \\
2016^{17}\end{array}$} & \multirow{2}{*}{ Retrospective } & PVP & 38 & $73 \pm 6$ & $20 / 18$ & \multirow{2}{*}{$B, C, D, F$} & \multirow{2}{*}{$\star \star \star \star \star$} \\
\hline & & PKP & 35 & $75 \pm 6$ & $16 / 19$ & & \\
\hline \multirow{2}{*}{ Li $2013^{18}$} & \multirow{2}{*}{ Retrospective } & PVP & 7 & \multirow{2}{*}{$71.8 \pm 7.69$} & $3 / 4$ & \multirow{2}{*}{$A, C, D, F$} & \multirow{2}{*}{$\star \star \star \star \star \star ~$} \\
\hline & & PKP & 5 & & $2 / 3$ & & \\
\hline \multirow{2}{*}{$\begin{array}{l}\text { Shi et al. } \\
2017^{19}\end{array}$} & \multirow{2}{*}{ Retrospective } & PVP & 10 & \multirow{2}{*}{$75.1 \pm 3.6$} & \multirow{2}{*}{$7 / 16$} & \multirow{2}{*}{$A, B, C, D, E, G$} & \multirow{2}{*}{$\star \star \star \star \star \star$} \\
\hline & & PKP & 13 & & & & \\
\hline \multirow{2}{*}{ Yu et al. $2016^{20}$} & \multirow{2}{*}{ Retrospective } & PVP & 14 & $74.47 \pm 5.79$ & $5 / 9$ & \multirow{2}{*}{$A, B, C, D, E, F$} & \multirow{2}{*}{$\star \star \star \star \star \star$} \\
\hline & & PKP & 28 & $71.56 \pm 8.35$ & $9 / 16$ & & \\
\hline \multirow{2}{*}{ Yu et al. $2016^{21}$} & \multirow{2}{*}{ Retrospective } & PVP & 48 & $74.6(63-85)$ & $10 / 38$ & \multirow{2}{*}{$A, B, C, D, E, F, G$} & \multirow{2}{*}{$\star \star \star \star \star \star$} \\
\hline & & PKP & 20 & $75.9(65-87)$ & $4 / 16$ & & \\
\hline \multirow{2}{*}{$\begin{array}{l}\text { Zhang et al. } \\
2015^{22}\end{array}$} & \multirow{2}{*}{ Retrospective } & PVP & 38 & $75.58 \pm 4.97$ & $10 / 28$ & \multirow{2}{*}{$B, D, E, F, G$} & \multirow{2}{*}{$\star \star \star \star \star \star$} \\
\hline & & PKP & 35 & $73.74 \pm 4.35$ & $9 / 26$ & & \\
\hline
\end{tabular}

Note: Outcomes: A: Cobb angle; B: Anterior vertebral body height; C: ODI; D: VAS score; E: Cement volume; F: Cement leakage; G: Operation time.

Table 1. General characteristics of included studies.

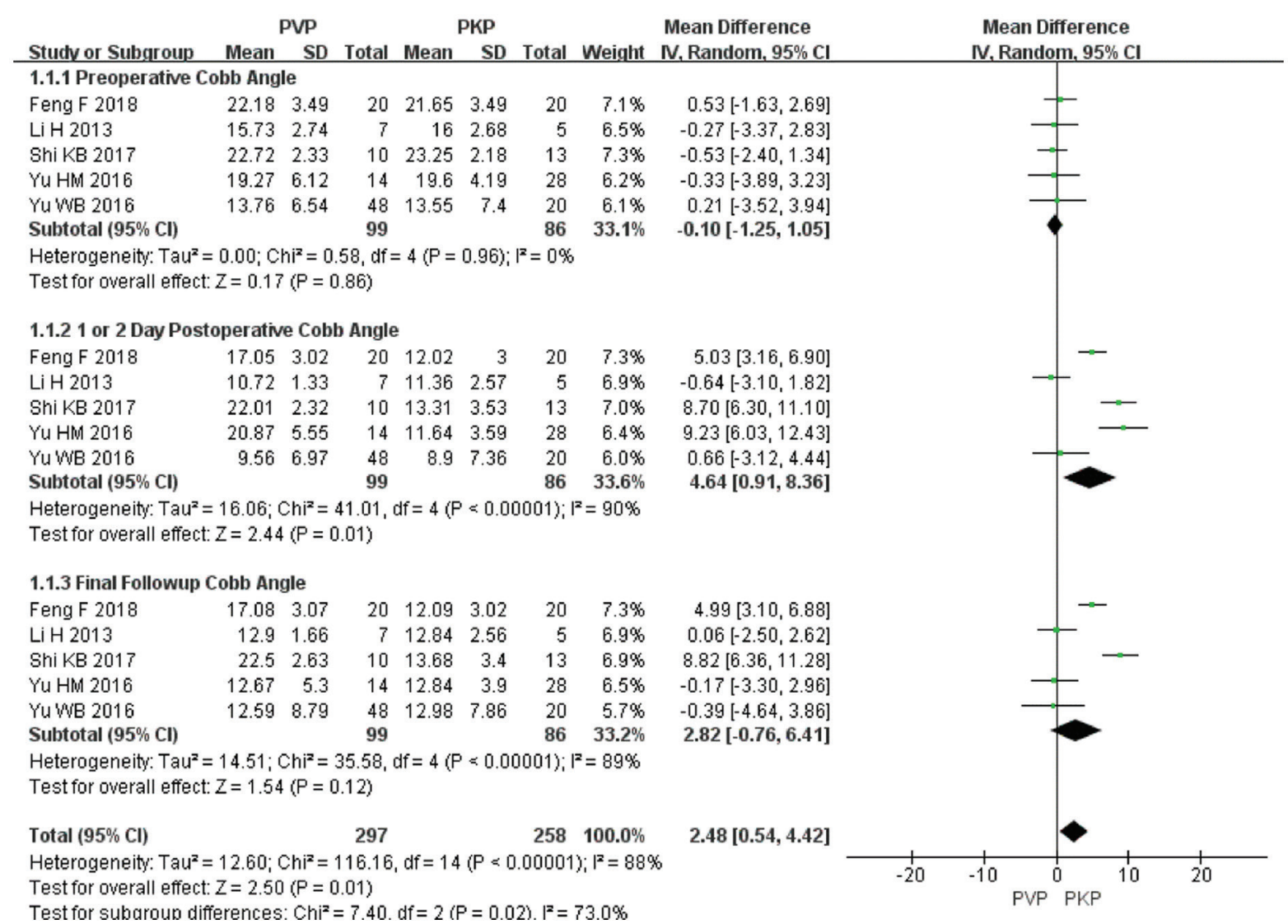

Figure 2. Forest plot to assess Cobb angle between two procedures 


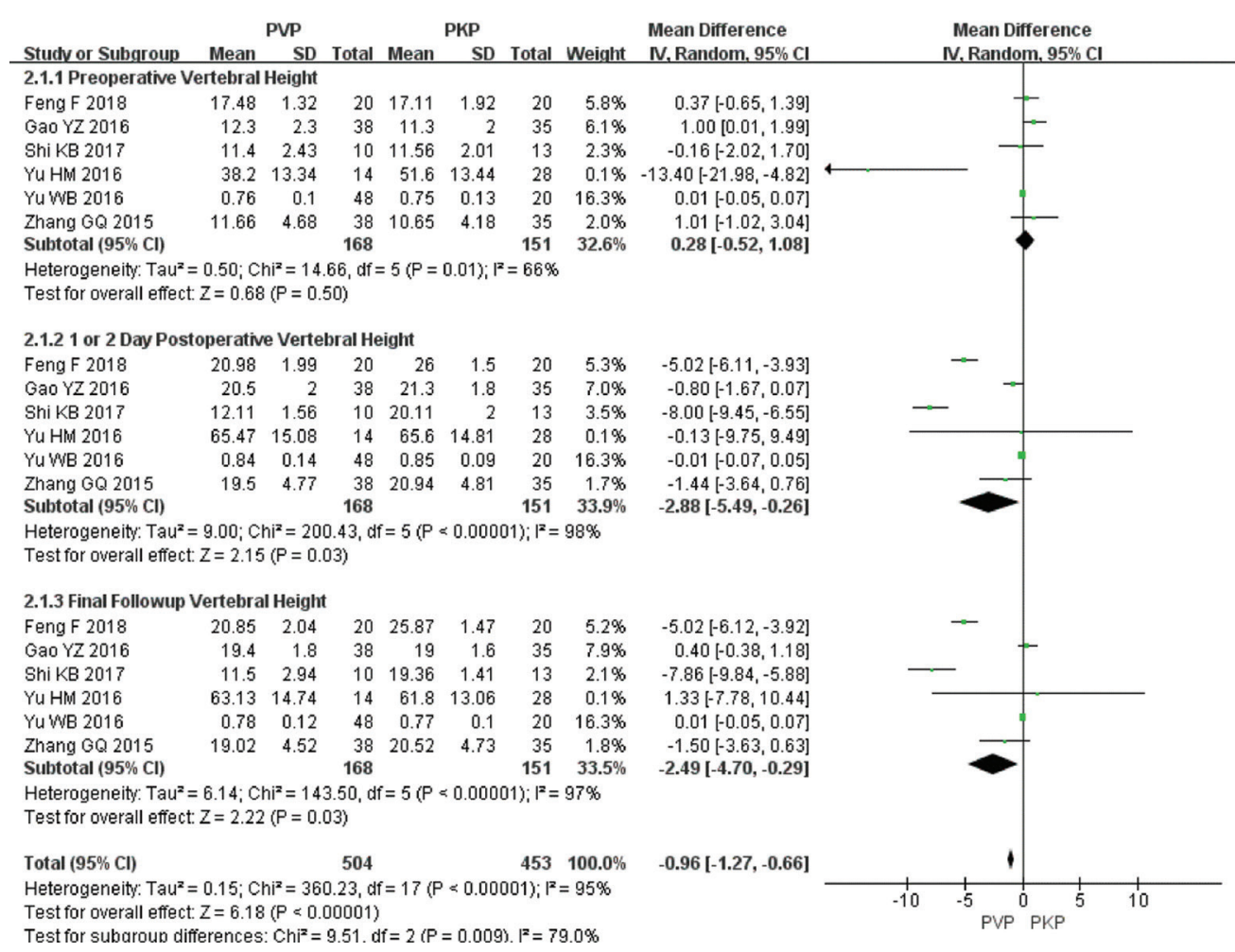

Figure 3. Forest plot to assess anterior vertebral body height between two procedures.

\begin{tabular}{|c|c|c|c|c|c|c|c|c|c|c|c|}
\hline \multirow[b]{2}{*}{ Studv or Subaroup } & \multicolumn{3}{|c|}{ PVP } & \multicolumn{2}{|c|}{ PKP } & & & Mean Difference & \multicolumn{3}{|c|}{ Mean Difference } \\
\hline & Mean & SD & Total & Mean & SD & Total & Weight & IV. Random. $95 \% \mathrm{Cl}$ & & IV. Random. 95\% & \\
\hline \multicolumn{12}{|c|}{ 3.1.1 Preoperative ODI } \\
\hline Feng F 2018 & 44.55 & 5.78 & 20 & 43.6 & 4.9 & 20 & $5.1 \%$ & $0.95[-2.37,4.27]$ & & & \\
\hline Gao YZ 2016 & 76.2 & 4.6 & 38 & 74 & 4.8 & 35 & $7.8 \%$ & $2.20[0.04,4.36]$ & & & \\
\hline LiH 2013 & 74.57 & 10.66 & 7 & 73.2 & 9.34 & 5 & $0.7 \%$ & $1.37[-10.00,12.74]$ & & & \\
\hline Shi KB 2017 & 73.7 & 3.14 & 10 & 74.63 & 6.8 & 13 & $3.8 \%$ & $-0.93[-5.11,3.25]$ & & & \\
\hline Yu HM 2016 & 78.08 & 3.2 & 14 & 72.97 & 4.07 & 28 & $7.5 \%$ & $5.11[2.86,7.36]$ & & & \\
\hline YUWB 2016 & 83.77 & 9.76 & 48 & 84.67 & 6.44 & 20 & $4.1 \%$ & $-0.90[-4.85,3.05]$ & & & \\
\hline Subtotal $(95 \% \mathrm{Cl})$ & & & 137 & & & 121 & $28.9 \%$ & $1.71[-0.41,3.84]$ & & & \\
\hline \multicolumn{12}{|c|}{$\begin{array}{l}\text { Heterogeneity: } \text { Tau }^{2}=3.56 ; \mathrm{Chi}^{2}=11.46, \mathrm{df}=5(\mathrm{P}=0.04) ; \mathrm{I}^{2}=56 \% \\
\text { Test for overall effect: } Z=1.58(\mathrm{P}=0.11)\end{array}$} \\
\hline \multicolumn{12}{|c|}{ 3.1.2 1 or 2 Day Postoperative ODI } \\
\hline Feng F 2018 & 28.95 & 5.97 & 20 & 27.65 & 3.9 & 20 & $5.5 \%$ & $1.30[-1.83,4.43]$ & & & \\
\hline Gao YZ 2016 & 20.2 & 2.7 & 38 & 19.1 & 2 & 35 & $11.0 \%$ & $1.10[0.02,2.18]$ & & & \\
\hline Li H 2013 & 19 & 3.16 & 7 & 18 & 2.55 & 5 & $5.2 \%$ & $1.00[-2.24,4.24]$ & & . & \\
\hline Shi KB 2017 & 42.83 & 6.75 & 10 & 42.14 & 6.63 & 13 & $2.5 \%$ & $0.69[-4.83,6.21]$ & & & \\
\hline Yu HM 2016 & 20.43 & 5.77 & 14 & 20.79 & 5.66 & 28 & $4.5 \%$ & $-0.36[-4.04,3.32]$ & & & \\
\hline YuWB 2016 & 27.3 & 7.4 & 48 & 28.33 & 7.97 & 20 & $3.9 \%$ & $-1.03[-5.10,3.04]$ & & & \\
\hline Subtotal $(95 \% \mathrm{Cl})$ & & & 137 & & & 121 & $32.5 \%$ & $0.90[-0.00,1.81]$ & & & \\
\hline \multicolumn{12}{|c|}{$\begin{array}{l}\text { Heterogeneity: } \mathrm{Tau}^{\mathrm{z}}=0.00 ; \mathrm{Ch}^{\mathrm{z}}=1.52, \mathrm{df}=5(\mathrm{P}=0.91) ; \mathrm{l}^{\mathbf{z}}=0 \% \\
\text { Test for overall effect: } Z=1.95(\mathrm{P}=0.05)\end{array}$} \\
\hline \multicolumn{12}{|c|}{ 3.1.3 Final Followup ODI } \\
\hline Feng F 2018 & 18.9 & 2.92 & 20 & 17.95 & 2.26 & 20 & $9.4 \%$ & $0.95[-0.67,2.57]$ & & 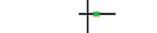 & \\
\hline Gao YZ 2016 & 18.3 & 2.5 & 38 & 17.5 & 1.6 & 35 & $11.3 \%$ & $0.80[-0.16,1.76]$ & & $=$ & \\
\hline Li H 2013 & 30.57 & 4.24 & 7 & 29 & 2 & 5 & $4.6 \%$ & $1.57[-2.03,5.17]$ & & & \\
\hline Shi KB 2017 & 45.7 & 8.72 & 10 & 30.34 & 7.18 & 13 & $1.8 \%$ & $15.36[8.69,22.03]$ & & & \\
\hline YU HM 2016 & 22.51 & 6.16 & 14 & 22.3 & 5.86 & 28 & $4.2 \%$ & $0.21[-3.68,4.10]$ & & & \\
\hline YU WB 2016 & 31.12 & 4.93 & 48 & 32.54 & 4.23 & 20 & $7.3 \%$ & $-1.42[-3.74,0.90]$ & & & \\
\hline Subtotal $(95 \% \mathrm{Cl})$ & & & 137 & & & 121 & $38.6 \%$ & $1.43[-0.60,3.46]$ & & & \\
\hline \multicolumn{12}{|c|}{$\begin{array}{l}\text { Heterogeneity: } \text { Tau }^{2}=4.18 ; \mathrm{ChF}^{2}=22.14, \mathrm{df}=5(P=0.0005) ; \mathrm{I}^{2}=77 \% \\
\text { Test for overall effect: } Z=1.38(P=0.17)\end{array}$} \\
\hline Total $(95 \% \mathrm{Cl})$ & & & 411 & & & 363 & $100.0 \%$ & $1.18[0.21,2.15]$ & & & \\
\hline \multicolumn{9}{|c|}{$\begin{array}{l}\text { Heterogeneity: } \mathrm{Tau}^{2}=1.91 ; \mathrm{Chi}^{2}=39.54, \mathrm{df}= \\
\text { Test for overall effect: } Z=2.39(\mathrm{P}=0.02) \\
\text { Test for subaroub differences: } \mathrm{Ch}^{2}=0.60 \text {. d }\end{array}$} & $\begin{array}{c}1 \\
-10\end{array}$ & $\begin{array}{lll}1 & 1 & 1 \\
-5 & 0 & 5 \\
\text { PVP PKP }\end{array}$ & 10 \\
\hline
\end{tabular}

Figure 4. Forest plot to assess ODI between the two procedures. 


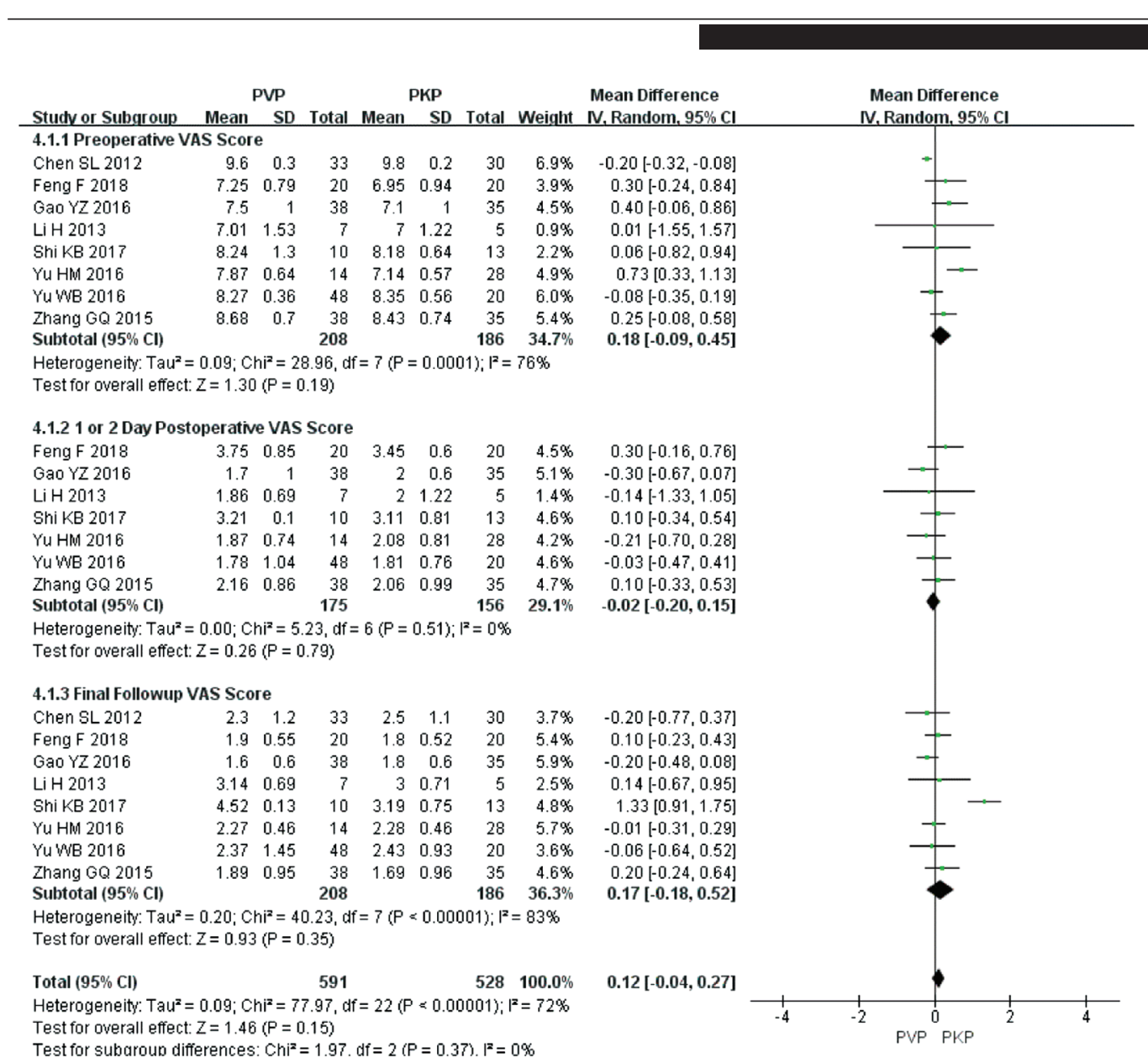

Test for subaroun differences: $\mathrm{Chi}^{2}=1.97 . \mathrm{df}=2(\mathrm{P}=0.37) . \mathrm{I}^{\mathrm{a}}=0 \%$

Figure 5. Forest plot to assess VAS score between the two procedures.

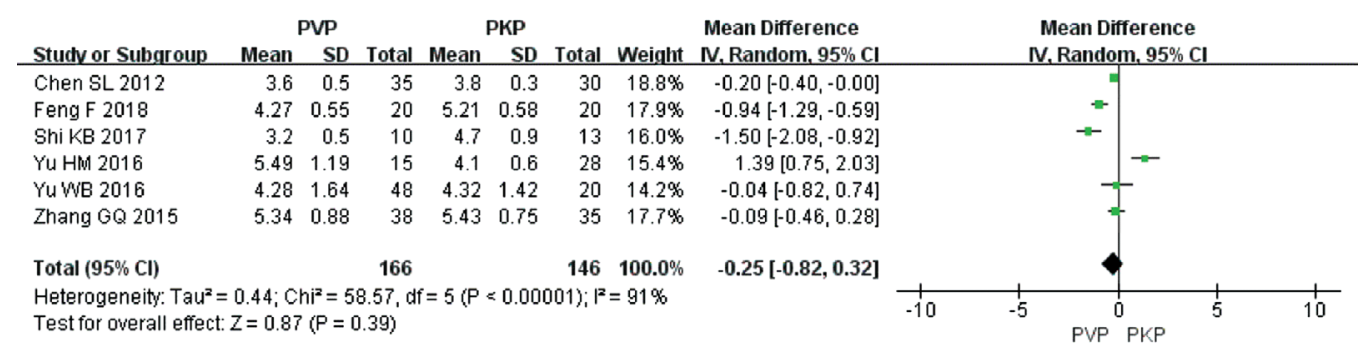

Figure 6. Forest plot to assess cement volume between two procedures.

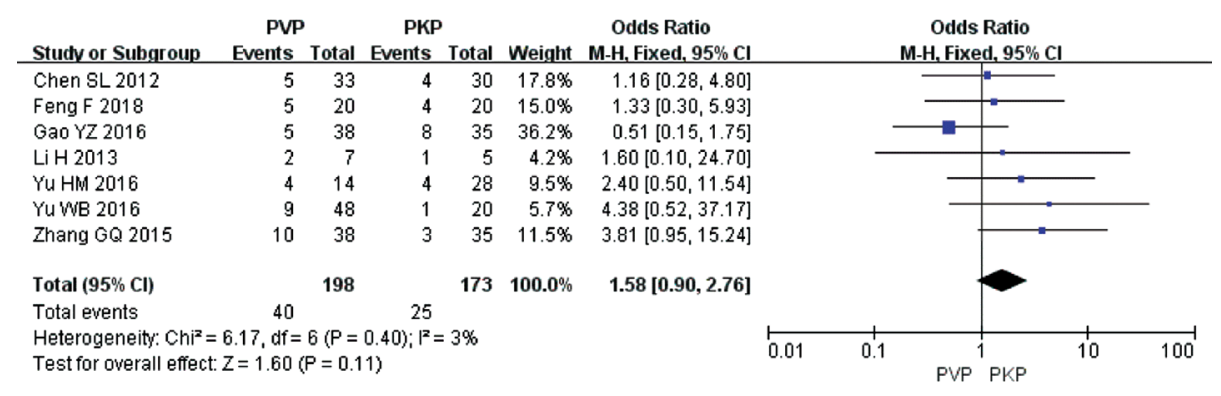

Figure 7. Forest plot to assess cases of cement leakage between the two procedures. 
time required for PVP group was less than that in PKP group (95\% Cl [-13.48, -7.43], $P<0.001$; Figure 8).

\subsection{Publication bias}

All the eight studies included in this meta-analysis had gone through a strict quality assessment. All of them were CCTs and the possibility of a bias was low. But the funnel figure showed that there was a small bias, which may be associated with the incomplete collection of relevant literature, insufficient sample size, and the different level of clinical physicians. Sensitivity analysis showed a good overall result (Figures 9 and 10).

Each risk of bias item is presented as a percentage across all included studies and indicates the proportional level for each risk of bias item.

Methodological quality of the included studies. This risk of bias tool incorporates assessment of randomization (sequence generation and allocation concealment), blinding (participants, personnel, and outcome assessors), completeness of outcome data, selection of outcomes reported, and other sources of bias. The items were scored with "yes," "no," or "unclear."

\section{Discussion}

Kümmell's disease is a special type of osteoporosis vertebra compressed fracture (OVCF), ${ }^{23}$ which accounts for about $10 \%$ of OVCF. ${ }^{24}$ It is reported few times due to the rare incidence and difficult diagnosis. However, as China enters an aging society with the explosion of the elderly population and advancement of imaging diagnostic technology, particularly the popularity of MRI has increased the reports of Kümmell's disease. ${ }^{25}$ The main symptoms of Kümmell's disease are back pain with or without spinal cord injury. ${ }^{26,27}$ The disease progresses progressively, and due to the obvious vertebral body necrosis and collapse, it can lead to severe kyphotic deformity in the late stage, which has a higher incidence of nerve injury than common OVCF. ${ }^{28,29} \mathrm{Li}$ et al. divided Kümmell's disease into three stages: in Stage I, vertebral body height reduction was $<20 \%$ without

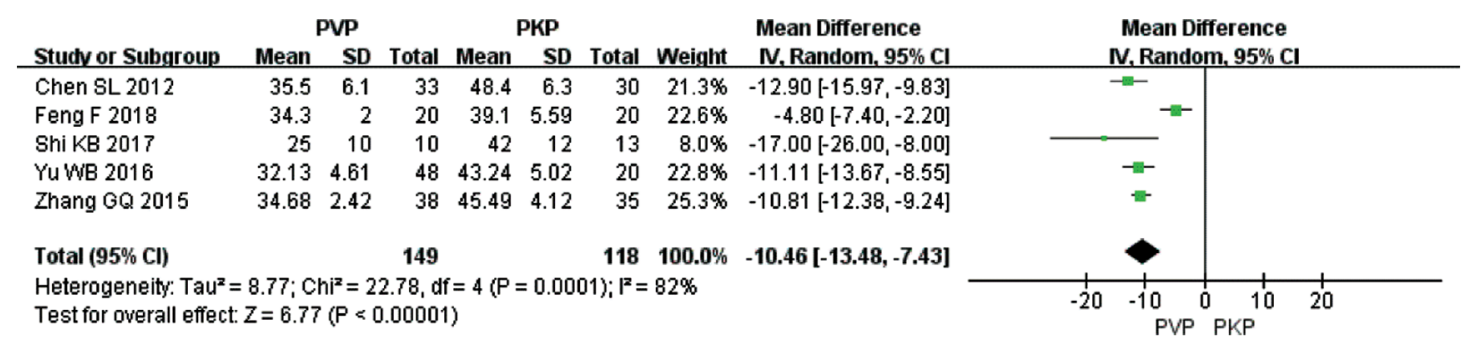

Figure 8. Forest plot to assess the operation time between the two procedures.

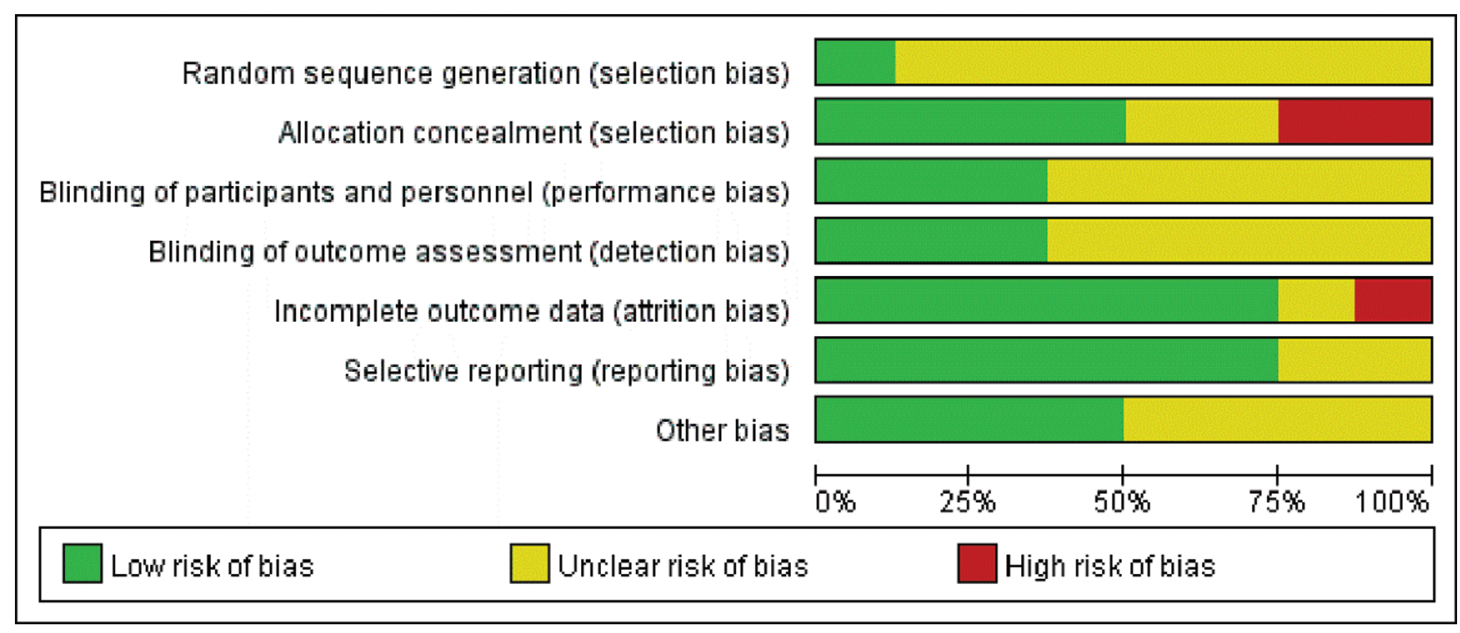

Figure 9. Risk of bias graph. 


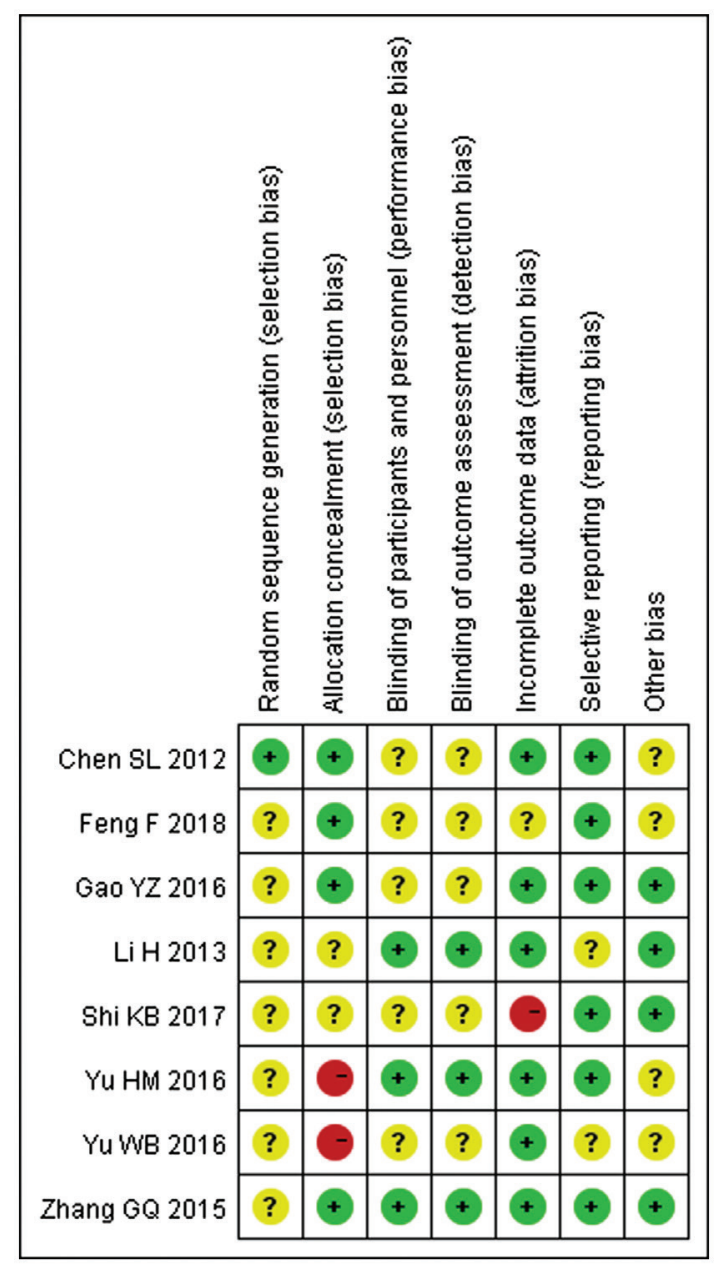

Figure 10. Risk of bias summary.

adjacent disc degeneration; in Stage II, vertebral body height reduction was $>20 \%$, often with adjacent disc degeneration; in Stage III, the posterior cortex of vertebrae ruptured with spinal cord compression. ${ }^{19}$ For stages I and II, PVP and PKP procedures are often used for vertebral bone cement treatment. ${ }^{30-32}$ But the vertebral body in stage III is incomplete due to posterior cortex collapse, and greater risk of cement leakage in vertebral canal exists during surgery, therefore open surgery is recommended. ${ }^{33,34}$ At present, PVP and PKP have become one of the most effective methods to treat vertebral tumors and $\mathrm{OVCF}^{35,36}$ (including Kümmell's disease).

The purpose of this meta-analysis was to compare the efficacy of PVP and PKP in the treatment of Kümmell's disease in Chinese patients. We selected seven outcomes including Cobb angle, vertebral body height, ODI, VAS score, amounts of cement injected, cement leakage, and operation time and strive to fully compare the efficacy between the two procedures. By comparison, Cobb angle $(95 \% \mathrm{Cl}[0.54,4.42], P=0.01)$, ODI (95\% Cl [0.21, 2.15], $P=0.02]$ and postoperative anterior vertebral body height $(95 \% \mathrm{Cl}[-1.27,-0.66], P<0.001)$ of the PKP group was better than PVP group and the difference was statistically significant. It indicates that there is no significant difference between the two groups in pain relief (VAS score), bone cement volume for injection and rate of leakage, but PKP is superior to PVP in terms of better functional improvement (ODI index), vertebral height recovery, and correction of kyphosis. However, the PVP group had less operation time than PKP group (95\% Cl [-13.48, -7.43], $P<0.001)$. PKP technology is an improved technology for PVP. By correcting kyphosis deformity through balloon dilation, bone cement can be injected at a lower pressure, but the operation time is longer than that of PVP due to the increase of operation steps. In summary, at this stage PKP has a clear edge over PVP in the treatment of Chinese Kümmell's disease at I and II stage. Although there is no evidence to support that PVP is invalid, in some hospitals with limited technical conditions, PVP is still an effective treatment for Kümmell's disease.

\section{Conclusions}

This systematic review included seven Chinese articles and one English article, and the methodological quality evaluation results were all high. Most studies were case-control studies. The purpose of this study is to compare the clinical efficacy of two cement augmentation procedures in Kümmell's disease treatment at I and II stages in Chinese patients. Therefore, the patient's informed consent, the choice of a specific treatment plan, the medical ethical issues, and the other inevitable bias also impact the reliability of the meta-analysis. In the same outcome measurements system, we include into the maximum of eight articles least of five and the heterogeneity between groups will increase. Therefore, the above conclusions still need to further verify depending on the emergence of RCTs with higher quality and larger sample sizes in the future.

\section{Ethical approval}

This study was approved by the ethics committee of Shanxi Medical University (IRB approval number: 201622083).

\section{Conflicts of interest}

All contributing authors declare no conflicts of interest. 


\section{References}

1. Li H, Liang CZ, Chen QX. Kummell's disease, an uncommon and complicated spinal disorder: a review. J Int Med Res. 2012;40:406-414.

2. Matzaroglou C, Georgiou CS, Panagopoulos A, et al. Kümmell's disease: clarifying the mechanisms and patients' inclusion criteria. Open Orthop J. 2014;8:288-297.

3. Lim J, Choi SW, Youm JY, Kwon HJ, Kim SH, Koh HS. Posttraumatic delayed vertebral collapse: Kummell's disease. J Korean Neurosurg Soc. 2018;61:1-9.

4. Matzaroglou C, Georgiou CS, Assimakopoulos K, Saridis A, Khudiaev AT, Giannakenas C. Kummell' s disease: a rare spine entity in a young adult. Hell $J$ Nucl Med. 2010;13:52-55.

5. Yu HM, Li YZ, Yao XD, et al. Surgical options for delayed osteoporotic vertebral collapse. China J Orthop Trauma. 2016;29:606-613 (in Chinese).

6. Zhang JN, Fan Y, He X, et al. Is percutaneous kyphoplasty the better choice for minimally invasive treatment of neurologically intact osteoporotic Kümmell's disease? A comparison of two minimally invasive procedures. Int Orthop. 2018;42:1321-1326.

7. Wang B, Zhao CP, Song LX, Zhu L. Balloon kyphoplasty versus percutaneous vertebroplasty for osteoporotic vertebral compression fracture: a meta-analysis and systematic review. J Orthop Surg Res. 2018;13:264.

8. Filippiadis DK, Marcia S, Masala S, Deschamps F, Kelekis A. Percutaneous vertebroplasty and kyphoplasty: current status, new developments and old controversies. Cardiovasc Intervent Radiol. 2017;40:1815-1823.

9. Kamei S, Noguchi T, Shida Y, et al. The safety and efficacy of percutaneous vertebroplasty for patients over 90 years old. Jpn J Radiol. 2019;37:178-185.

10. Rabei R, Patel K, Ginsburg M, et al. Percutaneous vertebral augmentation for vertebral compression fractures: national trends in the medicare population (2005-2015). Spine (Phila Pa 1976). 2019;44:123-133.

11. Yilmaz A, Cakir M, Yucetas CS, et al. Percutaneous kyphoplasty: is bilateral approach necessary? Spine (Phila Pa 1976). 2018;43:977-983.

12. Qi $Y$, Zeng $Y$, Jiang $C$, et al. Comparison of percutaneous kyphoplasty versus modified percutaneous kyphoplasty for treatment of osteoporotic vertebral compression fractures. World Neurosurg. 2019;122:e1020-e1027.
13. Park JW, Park JH, Jeon HJ, Lee JY, Cho BM, Park SH. Kümmell's disease treated with percutaneous vertebroplasty: minimum 1 year follow-up. Korean $J$ Neurotrauma. 2017;13:119-123.

14. Chen GD, Lu Q, Wang GL, et al. Percutaneous kyphoplasty for kummell disease with severe spinal canal stenosis. Pain Physician. 2015;18:E1021E1028.

15. Chen SL, Wang YS, Liu HJ, et al. Clinical results of percutaneous vertebroplasty and kyphoplasty for the treatment of osteoporotic Kummell's disease. Chin J Exp Surg. 2012;29:523-525 (in Chinese).

16. Feng F, Sun YL. A comparison of clinical efficacy of percutaneous vertebroplasty and percutaneous kyphoplasty in the treatment of Kümmell disease. Chin J Bone Joint. 2018;7:225-229 (in Chinese).

17. Gao YZ, Zhang GQ, Xing S, Chen SL, Ding S. Comparative analysis of percutaneous vertebroplasty and percutaneous kyphoplastyon in the treatment of the elderly with Kümmell disease. Chin J Geriatr Orthop Rehabil (Electronic Ed). 2016;2:136-140 (in Chinese).

18. Li H. A Comparison Study of Percutaneous Vertebraphasty and Percutaneous Kyphoplasty in the Treatment of Kummell's Disease. Zhejiang University; 2013. (in Chinese).

19. Shi KB, Chen Y, Xu H, Shi XJ. Vertebroplasty and balloon angioplasty Comparison of the effect of treating Kummell's disease without neurological symptoms. Med J Commun. 2017;31:3-5 (in Chinese).

20. Yu HM, Li YZ, Yao XD, et al. Percutaneous vertebroplasty or percutaneous kyphoplasty for Kummell's disease with vertebral posterior wall collapse: how to treat individually? Chin J Tissue Eng Res. 2016;20:3856-3862 (in Chinese).

21. Yu WB, Liang D, Jiang XB, Ye LQ, Yao ZS. Comparison of effectiveness between percutaneous vertebroplasty and percutaneous kyphoplasty for treatment of osteoporotic vertebral compression fracture with intravertebral vacuum cleft. Chin J Reparative Reconstr Surg. 2016;30:1104-1110 (in Chinese).

22. Zhang GQ, Gao YZ, Chen SL, Ding S, Gao K, Wang $H Q$. Comparison of percutaneous vertebroplasty and percutaneous kyphoplasty for the management of Kummell's disease: a retrospective study. Indian J Orthop. 2015;49:577-582. 
23. Matzaroglou C, Georgiou CS, Wilke HJ, et al. Kummell's disease: is ischemic necrosis or vertebral "microcracking" the first step in the sequence? Med Hypotheses. 2013;80:505.

24. Matzaroglou C, Georgiou CS, Assimakopoulos K, et al. Kummell's disease: pathophysiology, diagnosis, treatment and the role of nuclear medicine. Rationale according to our experience. Hell J Nucl Med. 2011;14:291-299.

25. Li H, Liang C, Shen C, Chen Q. Decreases in fluid shear stress due to microcracks: a possible primary pathogenesis of Kummell's disease. Med Hypotheses. 2011;77:897-899.

26. Kim HS, Lee SK, Kim SW, Shin H. Chronic spinal epidural hematoma related to Kummell's disease. J Korean Neurosurg Soc. 2011;49:231-233.

27. Wang D, Wang L, Xu J, Zen Y, Zheng S, Wang G. Value of forceps biopsy and kyphoplasty in Kummell's disease. Orthopedics. 2013;36:e1014-e1019.

28. Lee SH, Kim ES, Eoh W. Cement augmented anterior reconstruction with short posterior instrumentation: a less invasive surgical option for Kummell's disease with cord compression. J Clin Neurosci. 2011;18:509-514.

29. Ranjan M, Mahadevan A, Prasad C, Sommana S, Susarla Krishna S. Kummell's disease - uncommon or underreported disease: a clinicopathological account of a case and review of literature. J Neurosci Rural Pract. 2013;4:439-442.
30. Yao NZ, Xia X, Tang SF. Grded infusion of bone cement in percutaneous vertebroplasty for Kummell's disease. Chin J Reparative Reconstr Surg. 2015;29:1265-1268 (in Chinese).

31. Park SJ, Kim HS, Lee SK, Kim SW. Bone cementaugmented percutaneous short segment fixation: an effective treatment for Kummell's disease? J Korean Neurosurg Soc. 2015;58:54-59.

32. Zhong BY, He SC, Zhu H. Nomogram for predicting intradiscal cement leakage following percutaneous vertebroplasty in patients with osteoporotic related vertebral compression fractures. Pain Physician. 2017; 1:513-520.

33. D'Oria S, Delvecchio C, Dibenedetto M, Zizza F, Somma C. Case report of Kummell's disease with delayed onset myelopathy and the literature review. Eur J Orthop Surg Traumatol. 2018;28:309-316.

34. Cho Y. Corpectomy and circumferential fusion for advanced thoracolumbar Kummell's disease. Musculoskelet Surg. 2017;101:269-274.

35. Ren HL, Wang JX, Chen JT, Jiang JM. Clinical efficacy of unipedicular versus bipedicular percutaneous vertebroplasty for Kummell's disease. J South Med Univ. 2014;34:1370-1374 (in Chinese).

36. Ye LQ, Liang D, Jiang XB, et al. Risk factors for the occurrence of insufficient cement distribution in the fractured area after percutaneous vertebroplasty in osteoporotic vertebral compression fractures. Pain Physician. 2018;21:E33-E42. 